\title{
Evidence for in vitro and in vivo expression of the conserved VAR3 (type 3) plasmodium falciparum erythrocyte membrane protein 1
}

\author{
Christian W Wang ${ }^{1 *}$, Thomas Lavstsen', Dominique C Bengtsson ${ }^{1}$, Pamela A Magistrado', Sanne S Berger ${ }^{1}$, \\ Andrea M Marquard ${ }^{1}$, Michael Alifrangis ${ }^{1}$, John P Lusingu ${ }^{2}$, Thor G Theander $^{1}$ and Louise Turner $^{1}$
}

\begin{abstract}
Background: Members of the Plasmodium falciparum erythrocyte membrane protein 1 (PfEMP1) adhesion antigen family are major contributors to the pathogenesis of $P$. falciparum malaria infections. The PfEMP1-encoding var genes are among the most diverse sequences in nature, but three genes, var1, var2csa and var3 are found conserved in most parasite genomes. The most severe forms of malaria disease are caused by parasites expressing a subset of antigenically conserved PfEMP1 variants. Thus the ubiquitous and conserved VAR3 PFEMP1 is of particular interest to the research field. Evidence of VAR3 expression on the infected erythrocyte surface has never been presented, and var3 genes have been proposed to be transcribed and expressed differently from the rest of the var gene family members.
\end{abstract}

Methods: In this study, parasites expressing VAR3 PfEMP1 were generated using anti-VAR3 antibodies and the var transcript and PfEMP1 expression profiles of the generated parasites were investigated. The lgG reactivity by plasma from children living in malaria-endemic Tanzania was tested to parasites and recombinant VAR3 protein. Parasites from hospitalized children were isolated and the transcript level of var3 was investigated.

Results: Var3 is transcribed and its protein product expressed on the surface of infected erythrocytes. The VAR3-expressing parasites were better recognized by children's IgG than a parasite line expressing a Group B var gene. Two in 130 children showed increased recognition of parasites expressing VAR3 and to the recombinant VAR3 protein after a malaria episode and the isolated parasites showed high levels of var3 transcripts.

Conclusions: Collectively, the presented data suggest that var 3 is transcribed and its protein product expressed on the surface of infected erythrocytes in the same manner as seen for other var genes both in vitro and in vivo. Only very few children exhibit seroconversion to VAR3 following a malaria episode requiring hospitalization, supporting the previous conclusion drawn from var3 transcript analysis of parasites collected from children hospitalized with malaria, that VAR3 is not associated with severe anaemia or cerebral malaria syndromes in children.

\section{Background}

Plasmodium falciparum is a major cause of mortality and disease in sub-Saharan Africa. Immunity to malaria in areas with intense transmission is acquired during childhood as a broad repertoire of specific protective antibodies to parasite-derived polymorphic variant

\footnotetext{
*Correspondence: cwang@sund.ku.dk

${ }^{1}$ Centre for Medical Parasitology at Department of International Health, Immunology, and Microbiology, University of Copenhagen and at Department of Infectious Diseases, Copenhagen University Hospital (Rigshospitalet), 1014 Copenhagen, Denmark

Full list of author information is available at the end of the article
}

antigens present on the infected erythrocyte surface, develops [1-3]. Plasmodium falciparum erythrocyte membrane protein 1 (PfEMP1) is the best characterized variant surface antigen [4-6]. A single parasite express only one or a few variants at a time [7-11] as the parasite develops from ring-shaped early forms into trophozoite stages, simultaneously with the onset of adhesion and antigenicity of the infected erythrocyte [12]. Members of the PfEMP1 family mediate the cyto-adherence of infected erythrocytes to host receptors, allowing parasites to avoid splenic clearance and an immense sequence variation within the protein family has evolved to escape 
counteracting host antibody responses [13,14]. The PfEMP1 family is encoded by approximately $60 \mathrm{var}$ genes per parasite genome [6,15], which can be divided in two four groups A-E based on their 5 'upstream region $[16,17]$. Three var genes, var1 (UPSA), var2csa (USPE), and var3 (aka Type 3) (UPSA) are conserved in their full length in the global parasite population [18-22]. Apart from these, most parasite genomes contain a set of vars encoding more diverse domain cassettes not spanning the full length genes [23]. PfEMP1s are composed of multiple Duffy binding-like (DBL) and cysteine-rich inter-domain region (CIDR) domains. Among the PfEMP1s the VAR1, VAR2CSA and VAR3 variants have unique domain compositions void of CIDR domains. While VAR1 is particularly, long consisting of seven DBL domains and one CIDR, VAR3 and VAR2CSA distinguishes themselves by not containing any CIDR domains. In addition VAR3 is the smallest of the PfEMP1 proteins [15] and is the only PfEMP1 consisting of only two domains; DBL $\alpha / \zeta$ and DBLe [23]. Var3 genes have arisen from a recombination between a DBL $\zeta$-DBLe encoding sequence only found in the 3 'end of var exon1 and an N-terminal DBL 1 sequence. Sequence analysis has shown that only the DBL $\zeta$ DBLe part of VAR3, which is 99\% identical between VAR3 sequences, is unique to the protein sub-family [23].

VAR2CSA binds chondroitin sulphate A (CSA) in the placenta and facilitates the parasite sequestration causing pregnancy-associated malaria. Similarly, organ specific PfEMP1 mediated sequestration has been linked to severe malaria in children [24], and evidence indicates that a restricted and antigenically conserved subset of variant surface antigens causes the most severe malaria syndromes in children [25-32]. Thus, it is hypothesized that defined PfEMP1 subtypes confer specific adhesion phenotypes for the parasites [23]. Several studies have aimed to identify the var types expressed in severe malaria [29,30,33-36]. Most of these have relied on defining the most prevalent var mRNA species by PCR amplification and sequencing of a short 350 bp DBL $\alpha$ tag present in all var genes except var2csa and var3. As this approach does not capture var3 sequences, quantitative PCR has been deployed to investigate var3 transcript levels in patient samples [29,37]. The overall conclusion from these studies is that members of the UPSA var genes are associated with severe malaria in children.

Because VAR3 is conserved, belongs to group A PfEMP1 and has an unusual domain structure, this protein could play a particular important role for the parasite and in development of malaria disease. Malariaendemic populations has been shown to have acquired antibodies that react with recombinant VAR3 protein [38], suggesting that the protein is expressed and immunogenic during natural infections. However, quantitative PCR studies of var3 transcript levels in malaria patient samples have shown that var3 transcripts was not associated to any particular syndrome of severe malaria [37]. A formal demonstration of VAR3 on the surface of P. falciparum-infected erythrocytes has never been achieved and evidence presented by Epp et al. [39] suggests that var3 transcription may be regulated differently from the rest of the var genes and expressed in a non-mutually exclusive manner due to an atypical intron activity.

In an effort to unravel some of the mystery surrounding this PfEMP1, parasites of different genetic background were successfully manipulated to express VAR3 on the surface of the infected erythrocytes. Analysis of these parasites suggests that both var3 mRNA and VAR3 protein is expressed similar to other PfEMP1s. Furthermore, in this study, evidence for in vivo expression of VAR3 in malariasick children is presented.

\section{Methods}

Parasite culture, in vitro selection and synchronization

Plasmodium falciparum clones 3D7 and IT/FCR3 parasites were cultured in $\mathrm{O}$ Rh + erythrocytes in RPMI1640 supplemented with Albumax II, as previously described [40]. Parasites were synchronized by magnet-activated cell sorting (MACS; Miltenyi BioTec) and $24 \mathrm{~h}$ later ring stage parasites were harvested for RNA-extraction. The developmental stage of parasites was confirmed by assessment of 500 erythrocytes on Giemsa-stained thin smears. Parasite cultures were centrifuged and $1 \mathrm{ml}$ Trizol (Invitrogen) was added to the pellet to preserve RNA. 3D7 and IT/FCR3 parasites were selected as previously described [41] by repeated rounds of panning on DynaBeads coated with rabbit IgG specific for the 3D7 VAR3 double domain of PFI1820w. A more stringent synchronization of the 3D7VAR3 line was done by gelatine purification of latestage trophozoites and schizonts and further enriched $48 \mathrm{~h}$ later on a MACS column. The synchronous population of parasites was cultured in six different culture flasks and harvested at six different time points of the intraerythrocytic life cycle. The developmental stages of the 3D7VAR3 parasites at the six time points were determined by assessment of 900 erythrocytes on thin smears stained by Giemsa.

Isolate integrity of the cultures was tested using the method previously described by Snounou et al. [42] with the following modifications: only the second round of amplification was done using the MSP2-IC1, MSP2FC27 and GLURP specific primer pairs. The conditions of the PCR were as follows for all three reactions: $94^{\circ} \mathrm{C}$ for $15 \mathrm{~min}, 35 \mathrm{cycles}$ of $94^{\circ} \mathrm{C}$ for $1 \mathrm{~min}, 61^{\circ} \mathrm{C}$ for $2 \mathrm{~min}$ and $65^{\circ} \mathrm{C}$ for $2 \mathrm{~min}$, followed by $58^{\circ} \mathrm{C}$ for $2 \mathrm{~min}$ and $65^{\circ} \mathrm{C}$ for $5 \mathrm{~min}$. The PCR reaction contained: $0.125 \mu \mathrm{M}$ of the primer pairs, 1:1 of TEMPase Hot Start DNA Polymerase (Ampliqon, VWR) and $1 \mu \mathrm{L}$ of DNA extracted using the DNeasy Kit (Qiagen) according to the manufacturer's 
instructions. A set of most common in-house used laboratory isolates (3D7, IT/FCR3, DD2, K1, and HB3) as well as $P$. falciparum negative controls, human DNA from Danish donors and blank samples, were included in the setup and the amplified PCR products were analysed by agarose gel electrophoresis.

\section{RNA extraction and CDNA synthesis}

RNA was extracted using Trizol (Invitrogen) according to manufacturer's instructions. Samples were treated with DNase I (Sigma) to digest any genomic DNA and tested in quantitative PCR for contamination, using a primer set, p90, for the seryl-tRNA synthetase gene. RNA was reverse transcribed from random hexamers, using Superscript II (Invitrogen), according to the manufacturer's instructions (Invitrogen).

\section{Primer design}

Quantitative primers for each var gene of the P. falciparum clone 3D7 and to the endogenous control genes seryltRNA synthetase and fructose-bisphosphate aldolase, have been described previously [20] with modifications [43]. Specific quantitative primers were designed for $56 \mathrm{var}$ genes extracted from the IT/FCR3 genome available at The Wellcome Trust Sanger Institute (UK) [44] (Additional file 1). The primers were validated by testing amplification efficiency relative to p90 on 10fold dilutions of genomic DNA using quantitative PCR. All primer pairs had amplification efficiencies $>90 \%$, and produced single band products on agarose gel electrophoresis with single melting peaks in melting curve analysis. Two cross-intron primers to detect spliced PFI1820w/PFF0020c/IT4var3 (var3Xi) and PFA0015c (PFA0015cXi) transcripts, respectively, were designed as well (Additional file 1). The amplification efficiency of these primers was not determined; however, their correct PCR amplification product sizes from genomic DNA and cDNA of both 3D7 and IT/FCR3 parasites was confirmed on agarose gel electrophoresis. A primer pair designed to universally amplify var3 transcripts was also used (Additional file 1). To further validate the specificity of all primers, the PCR products were sequenced, using the specific forward and reverse primer. All primer pairs were specific for their target genes.

\section{Quantitative PCR}

Quantitative PCR was performed on a Rotorgene RG-3000 thermal cycler (Corbett Research), applying QuantiTect SYBR Green PCR Master Mix (Qiagen) with primers at $20 \mu \mathrm{M}$, and internal control genes seryl-tRNA synthetase and fructose-bisphosphate aldolase, used for normalization. Gene-specific standard curves were produced by determining the amplification efficiency relative to the single copy internal control gene, seryl-tRNA synthetase, based on quantitative measurements of 10-fold dilutions of genomic DNA and used to calculate the transcript copy number of each gene in tested cDNA. Transcript levels relative to the average level of the two internal control genes were calculated as $2^{-\Delta \mathrm{Ct}(\text { var gene) }}$ and the level of internal control genes were set to 100 (User Bulletin \#2: ABI Prism 7700 Sequence Detection System; Applied Biosystems)

\section{Protein expression and generation of antiserum}

Protein expression was performed as previously described $[28,46]$ and the rabbit antiserum raised against recombinant DBL $\alpha$-DBLe double domain $(\sim 90 \mathrm{kDa})$ of the clone 3D7 PFI1820w protein from a previous study [38] was used in this present study. The antiserum was tested positive in ELISA for reactivity against the immunizing antigen [38]. Depletion of antibodies reacting with erythrocyte antigens was done by mixing equal amounts of antiserum with human $\mathrm{O}^{+}$erythrocytes and incubating $24 \mathrm{~h}$ at $4^{\circ} \mathrm{C}$. Besides the full length VAR3 protein, two full length and four multi-domain PfEMP1 proteins were included in the Luminex assay (Additional file 2). The C-terminal region of MSP3 was included as control as previously described [48].

\section{Flow cytometry}

The surface expression of VAR3 PfEMP1 by 3D7VAR3 and FCR3VAR3 lines was assessed by flow cytometry [49]. The erythrocyte surface antigen phenotype of line FCR3VAR3 was characterized using plasma from 61 children (from age one to 19 years) living in Mgome, Tanzania (an area of holo-endemic malaria transmission), from a cross-sectional survey [50], for IgG surface-staining of infected erythrocytes. Danish plasma was used as a control. Acute and convalescent plasma from 130 under five-yearold children living in north-eastern Tanzania experiencing a malaria episode was also tested with the FCR3VAR3 line. Informed consent forms were signed by parent or legal guardians of all subjects and the trials were reviewed and approved by the Medical Research Coordinating Committee, Tanzania (NIMR/HQ/R.8a/Vol.IX/559). The data was acquired using a FC500 instrument (Beckman Coulter). Uninfected erythrocytes were analysed to exclude IgG binding to erythrocyte antigens.

\section{Confocal microscopy}

Indirect immuno-fluorescence assays were performed on live 3D7VAR3/3D7 unselected and FCR3VAR3/IT/FCR3 unselected in order to observe the staining of individual infected erythrocytes with VAR3-antibodies. The staining was done using Alexa ${ }^{\mathrm{TM}} 488$ anti-rabbit IgG (Invitrogen) and DAPI and laser scanning confocal microscopy performed using a Nikon TE 2000-E confocal microscope with 60x oil immersion objective lens (DIC) as previously described [51]. 


\section{Luminex}

Levels of plasma PfEMP1 domain-specific IgG were analysed as described previously [52]. In brief, lyophilized microspheres were reconstituted with distilled water immediately prior to use and diluted 1:333 in assay buffer $\mathrm{E}$ (ABE; 0.1\% bovine serum albumin [BSA], 0.05\% Tween 20 , and $0.05 \%$ sodium azide in PBS, $\mathrm{pH} 7.4$ ). Aliquots $(50 \mu \mathrm{l})$ were dispensed into the wells of $1.2-\mu \mathrm{m}$ filter-bottom 96-well microtitre plates (MSBVS 1210; Millipore) prewetted with $\mathrm{ABE}$ and washed three times with $\mathrm{ABE}$ by using a vacuum manifold (Millipore). Frozen plasma samples were thawed at room temperature, mixed by vortexing, and spun $(16,000 \times \mathrm{g}$ for $5 \mathrm{~min})$ to remove particulates. Plasma samples were diluted 1:80 in ABE and 50- $\mu$ l aliquots were added to the microsphere wells. After incubation in the dark on a shaking platform (30 sec at 1,100 rpm followed by $30 \mathrm{~min}$ at $300 \mathrm{rpm}$ ), the plates were washed three times in $\mathrm{ABE}$ to remove unbound antibody. Biotinylated anti-human IgG (Sigma) antibody (25 $\mu \mathrm{l} /$ well at 1:500 dilution) was added to the microspheres, which were incubated and washed as described above. This was followed by use of streptavidin-phycoerythrin (Sigma) (50 $\mu \mathrm{l} /$ well at 1:500), incubation in the dark with shaking (30 sec at 1,100 rpm followed by $10 \mathrm{~min}$ at $300 \mathrm{rpm}$ ), and washing as described above. Finally, the microspheres were resuspended in $125 \mu \mathrm{l}$ of $\mathrm{ABE}$ and analysed on a Luminex 100 IS instrument set to read a minimum of 100 microspheres per microsphere region. Antibody levels for each domain were expressed as median fluorescence intensities (MFI). A negative cut-off value of 249 MFI units, based on the reactivity measured in plasma from unexposed control donors was used.

\section{Results}

Generation of VAR3 expressing 3D7 and IT/FCR3 parasite lines

To generate in vitro model parasite lines expressing VAR3, parasites of two laboratory cultured parasite clones, 3D7 and IT/FCR3, were selected with rabbit antiserum against a 3D7 VAR3 (PFI1820w) recombinant protein. The expression of VAR3 on the surface of 3D7VAR3- and FCR3VAR3infected erythrocytes, respectively, was investigated by flow cytometry and confocal microscopy using rabbit IgG specific for the clone 3D7 VAR3 PFI1820w which they had been selected with. The selected lines, 3D7VAR3 and FCR3VAR3, were recognized by the rabbit serum and stained the infected erythrocytes in a punctuate pattern typical for PfEMP1 [11,51] whereas the unselected lines were not recognized (Figure 1). Non-immune rabbit IgG did not stain the infected erythrocytes.

VAR3 expressing parasite lines exhibit transcript patterns similar to other var gene expressing parasites

The var transcript profiles of the selected 3D7VAR3 and FCR3VAR3 and unselected 3D7 and IT/FCR3 parasite lines were investigated using var gene specific primers in quantitative PCR. The transcript levels of all var genes relative to internal control genes are shown in Figure 2. The selected lines, 3D7VAR3 and FCR3VAR3, showed markedly higher levels of var3 transcripts, PFI1820w and IT4var3, than the unselected lines and the var3 transcript levels were similar to those observed for var genes in vivo [53] and other adhesion-selected in-vitro parasite cultures with verified erythrocyte surface expression of PfEMP1 [20,28]. Interestingly, antibody selection of 3D7, which harbour three var3 genes, resulted in selection of parasites expressing two of these genes (PFI1820w and $P F F 0020 c)$ but not parasites expressing the third (PFA0015c) (Figure 2a). A specific cross-intron primer pair confirmed the correct splicing of var3 transcripts (Table 1). To investigate whether the pattern of var3 transcription diverge from that of other var genes, the 3D7VAR3 line was synchronized and RNA isolated throughout the intra-erythrocytic asexual lifecycle. The two var3 genes, PFI1820w and PFF0020c, were the dominant var transcripts during the entire cycle with the exception at $0 \mathrm{~h}$ post MACS (PM), with a peak in transcript level at $19 \mathrm{~h} \mathrm{PM}$ corresponding to mid ring stages (Figure 3) and thus exhibits a transcription pattern similar to var2csa in a VAR2CSA-expressing parasite line [43] and four group A var genes in the 3D7-Lib parasite line [45].

\section{Serorecognition of 61 plasma samples from Mgome of FCR3VAR3}

The IgG reactivity to live FCR3VAR3 parasites in plasma from 61 individuals between one and 19 years of age living in a high transmission village of Mgome, Muheza Tanzania, was evaluated by flow cytometry (Figure 4). The 61 individuals were split into five different age groups (one to three, three to five, five to 10,10 to 15 , 15 to 19) and eight Danish donors were used as controls. The level of reactivity was compared to that of parasites expressing a Group A var gene, IT4var60, thought to mediate rosetting [54] and parasites expressing a Group B var gene, IT4var59 (Additional file 3). The domain structures of the three expressed PfEMP1 proteins are shown as well (Figure 4). The FCR3VAR3 parasites were recognized by similar levels of IgG in children's plasma as the parasites expressing IT4var60 and by higher levels of IgG than the parasites expressing group B IT4var59 (Figure 4). For the var3 and IT4var60 expressing parasite lines the $<3$ year olds showed the lowest IgG level but the level rose to a plateau already at the age of 3 to $<5$ years old. All 61 children had higher IgG levels against these two parasite lines than the Danish control donors. In contrast, the IT4var59 parasite line was only recognized by a few of the Mgome plasma donors with a peak in the group of 10 to 15 year olds. 


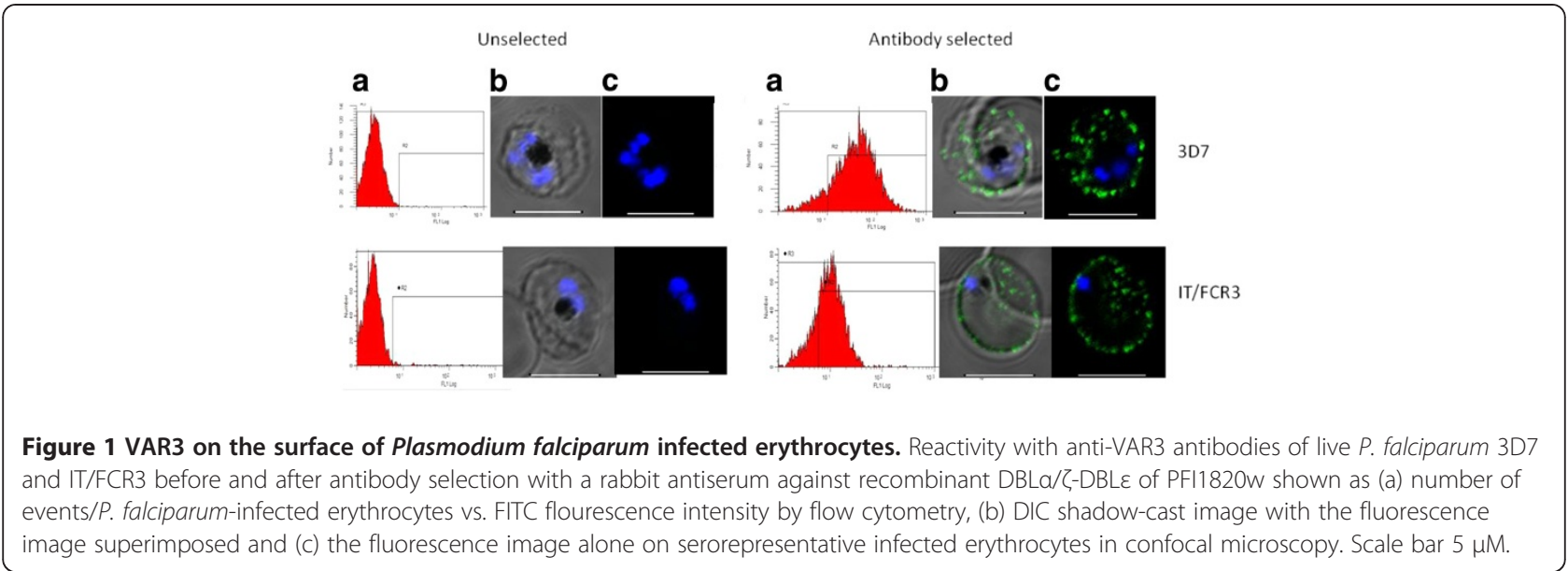

\section{Acute-convalescent plasma pairs and patient parasite isolates}

The IgG reactivity to the FCR3VAR3 parasite line and recombinant VAR3 protein was investigated in acute and convalescent plasma from 130 children under five years of age from high malaria transmission villages of Korogwe, Tanzania, experiencing a malaria episode. Antibodies to FCR3VAR3 was observed in 15 (11\%) of the acute patient plasma samples. Only two boys, patient A and B, appeared to acquire IgG to VAR3 but not to VAR2CSA expressing parasites after being sick and hospitalized with malaria (Figure 5a and b). An increased level of VAR3 IgG in patient $\mathrm{A}$ and $\mathrm{B}$ convalescent plasma was also found by luminex assay assessment of IgG levels to recombinant full length VAR3 and a panel of six different multi-domain and full length PfEMP1 proteins (Figure $5 \mathrm{c}$ and d). Patient A and $\mathrm{B}$ were hospitalized in north-eastern Tanzania at the age of $2^{2} / 3$ and 5 years old with body temperatures of $39.5^{\circ}$ $\mathrm{C}$ and $39.8^{\circ} \mathrm{C}$, parasitaemias of 266,735 and 159,470 parasites $/ \mu \mathrm{L}$, Blantyre Coma Scores of 5 , lactate levels of $3 \mathrm{mM}$, and haemoglobin values of 8.6 and $10.8 \mathrm{~g} / \mathrm{dl}$, respectively. Patient B appeared to have a broader antiPfEMP1 IgG repertoire than patient $A$, which could be due to a difference in exposure as patient $B$ is almost twice the age of patient A. Total RNA from the infecting parasites was isolated and the level of var3 transcripts was investigated using universal and 3D7 and IT/FCR3 var3 specific primers in quantitative PCR. The var3 transcript levels in these samples were lower than in the VAR3selected laboratory parasite lines (Table 1), but at levels previously considered high in var transcript analyses of field samples (Additional file 4). The transcript levels of other var genes in the samples were assessed using a set of 42 var subtyping quantitative PCR primers [37] (Additional file 4). These data indicates that apart from var3, other group A and DC8 encoding var genes are expressed at high levels in the two patients.

\section{Discussion}

The PfEMP1 mediated parasite sequestration causes harmful inflammation and severe pathology of malaria disease and evidence suggests that particular PfEMP1 subtypes are associated with different syndromes of malaria (reviewed in [55]). In pregnant women, malaria is caused by VAR2CSA mediated parasite sequestration in the placenta [53] and the subgroup of UPSA flanked var genes which includes the conserved var3 variant has been associated with severe malaria in children [28-32]. The particular involvement of VAR2CSA in pregnancy malaria and the ubiquitous and conserved nature of VAR2CSA make it an apparent vaccine target. The same might apply for VAR3, but no particular function has been ascribed to VAR3 and analysis of var3 transcripts in patient isolates $[29,37]$ does not predict a particular association with severe malarial disease in children. Nevertheless, the data presented in this paper indicate that VAR3 expressing parasites are viable in vivo and can bestow significant proportions of parasite populations in children sick from malaria. Specifically, IgG from children down to the age of one year reacted with VAR3 PfEMP1expressing parasites where the level of recognition seemed to increase with age and find a plateau within the first three years of age. The VAR3-expressing parasites were better recognized by the children's IgG than a parasite line expressing a Group B var gene. Although VAR3 is less polymorphic than Group B PfEMP1s, these data may indicate an earlier exposure to VAR3-expressing parasites than to Group B PfEMP1-expressing parasites, suggesting a particular involvement in early childhood malaria as predicted for the UPSA type PfEMP1 in general [17,28-30]. Of the 130 acute-convalescent samples from children under five years of age, only two children were found to acquire IgG antibodies to VAR3 as a result of an acute malaria episode requiring hospitalization. Patient A suffered from hyperparasitaemia but neither of the two 

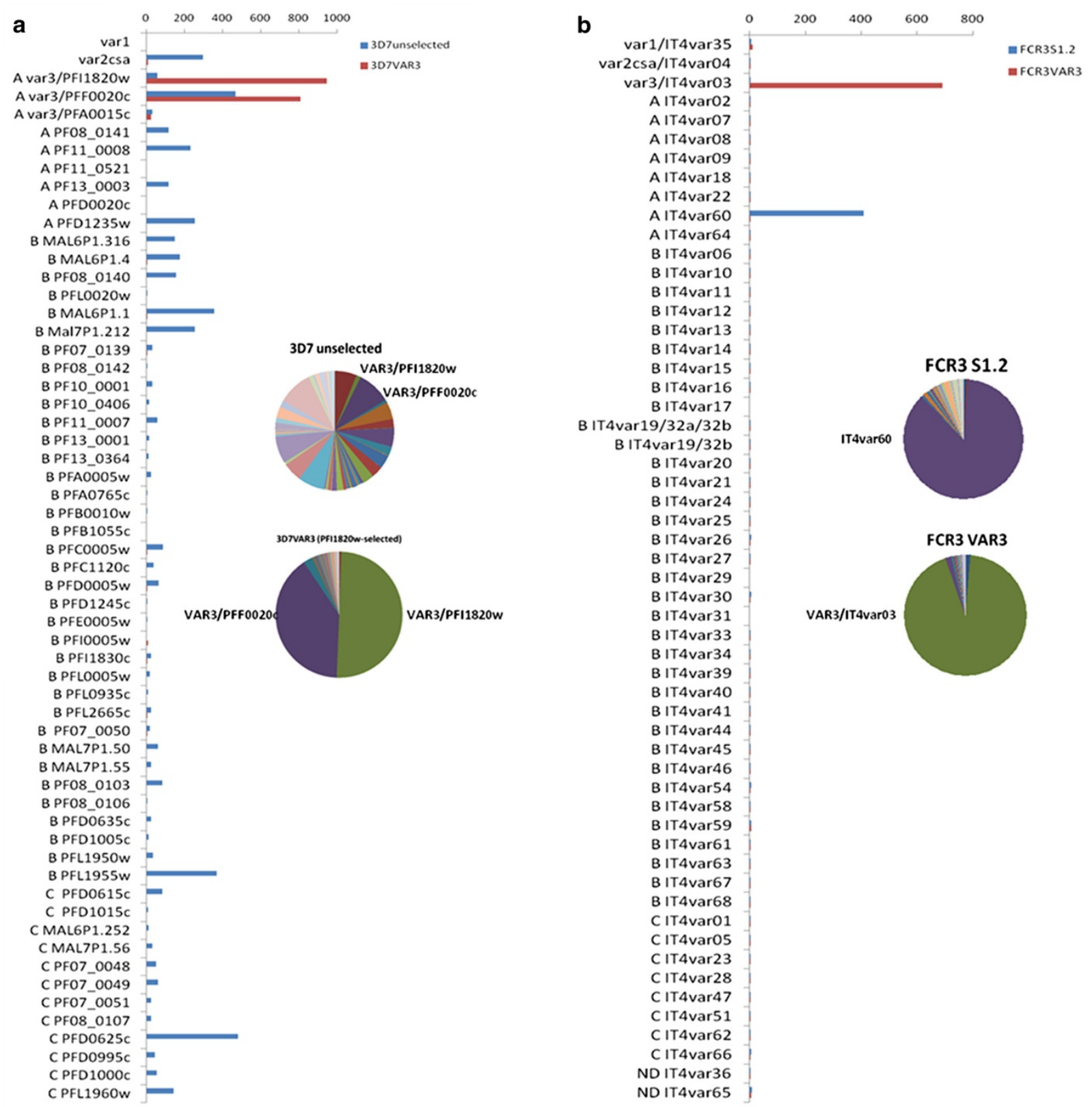

Figure 2 Var gene transcript levels in VAR3-expressing Plasmodium falciparum parasites. Var gene transcript levels relative to the average of two internal control genes before and after antibody selection of $P$. falciparum (a) 3D7 and (b) IT/FCR3 parasites, using Dynabeads coated with rabbit lgG specific for VAR3 PfEMP1. The average of control gene transcript levels is set to 100. The distribution of var transcripts before and after selection is also shown (circular diagrams). Transcript levels were measured by quantitative PCR using a set of primers that amplify 59 and 56 var genes from clone 3D7 and IT/FCR3, respectively. A cross-intron primer specific for the clone 3D7 var3 genes, PFI1820W and PFF0020c, and the clone IT/FCR3 var3 gene, IT4var03, showed similar transcript level as the corresponding exon 1 primer pairs (Table 1). The grouping of var genes are as previously described $[16,17,23]$.

patients was anaemic nor showed signs of cerebral malaria. Parasites isolated at hospitalization were found to transcribe var3-like genes at high levels, which together with the acquired antibodies indicate that VAR3-expressing parasites have contributed to the development of malaria symptoms in these patients. However, other var type transcripts were also detected in these patients and thus the VAR3-expressing parasites did not appear to be the only cause of disease in these two patients.

The pattern of Tanzanian children's acquisition of antibodies to live VAR3-expressing parasites compared to UPSA and UPSB type PfEMP1-expressing parasites 
Table 1 The RNA levels of var3 in patient A and B parasite isolates and four laboratory parasite lines

\begin{tabular}{lllllll}
\hline Sample Primer pair & Patient A & Patient B & 3D7 VAR3 & FCR3 VAR3 & FCR3 IT4var60 & FCR3 IT4var59 \\
\hline DBL88 (var3Global) & 187.6 & 419.9 & 1837.9 & 701.3 & 0.9 & 0.3 \\
PFI1820w (3D7) & ND & ND & 1055.6 & ND & ND & ND \\
PFF0020w (3D7) & ND & ND & 807.8 & ND & ND & ND \\
PFA0015c (3D7) & ND & ND & 17.7 & ND & ND & ND \\
IT4var3 (FCR3) & ND & ND & ND & 696.4 & 0.3 & 0.1 \\
VAR3Xi (3D7 and FCR3) & ND & ND & 1492.9 & 621.2 & 0.7 & 0.0 \\
PFA0015CXi (3D7) & ND & ND & 17.7 & 0.0 & 0.0 & 0.0 \\
\hline
\end{tabular}

RNA level is shown relatively to an average RNA level of the housekeeping genes, seryl-tRNA synthetase and fructose-bisphosphate aldolase, set to 100. ND: Not done.

indicate a virulence of VAR3 similar to UPSA type PfEMP1. Yet, in spite of the conserved nature of var3 and the presence in almost every parasite genome, it is striking that only a fifth of 1,342 individuals from malaria-endemic areas in Tanzania showed acquisition of antibodies to this PfEMP1 [38]. This may reflect either that VAR3 is not very immunogenic and/or parasites expressing VAR3 are not very virulent as also suggested by QPCR studies [29,37] and the acute/convalescent plasma study described here.

These interpretations of var3 transcribing parasites and VAR3 antibodies in malaria patients can be challenged if var3 genes are regulated and expressed abnormally as appears to be the case for var1. Studies of laboratory parasite lines show that var1 is constitutively transcribed throughout the blood stages cycle, while the transcription of other var genes is silenced when parasites develop into schizonts [56]. In spite of this and the fact that malaria endemic populations develop anti-
VAR1 antibodies [38], VAR1 erythrocyte surface expression has not been demonstrated. Analysis of available sequenced parasite genome shows that var1 often lacks or has a truncated exon2 which may cause a corrupted transcription regulation resulting in protein synthesis and host antibody development upon parasite destruction. It remains unclear if VAR1 serves a particular function for the parasite and why var1 is maintained in the population. Indeed, Epp and colleagues [39] found that the atypical var intron of the PFF0020c var3 gene did not show any transcriptional activity as opposed to other non-VAR3 var introns. Non-coding RNA is transcribed from var introns and is physically associated with chromatin and believed to be involved in the transcriptional regulation of var genes [39]. Thus, it was speculated that var3 genes in general could be regulated differently from other var genes. From the present data it does not seem that var3 genes are transcribed and expressed differently from other var genes. In both 3D7VAR3 and FCR3VAR3

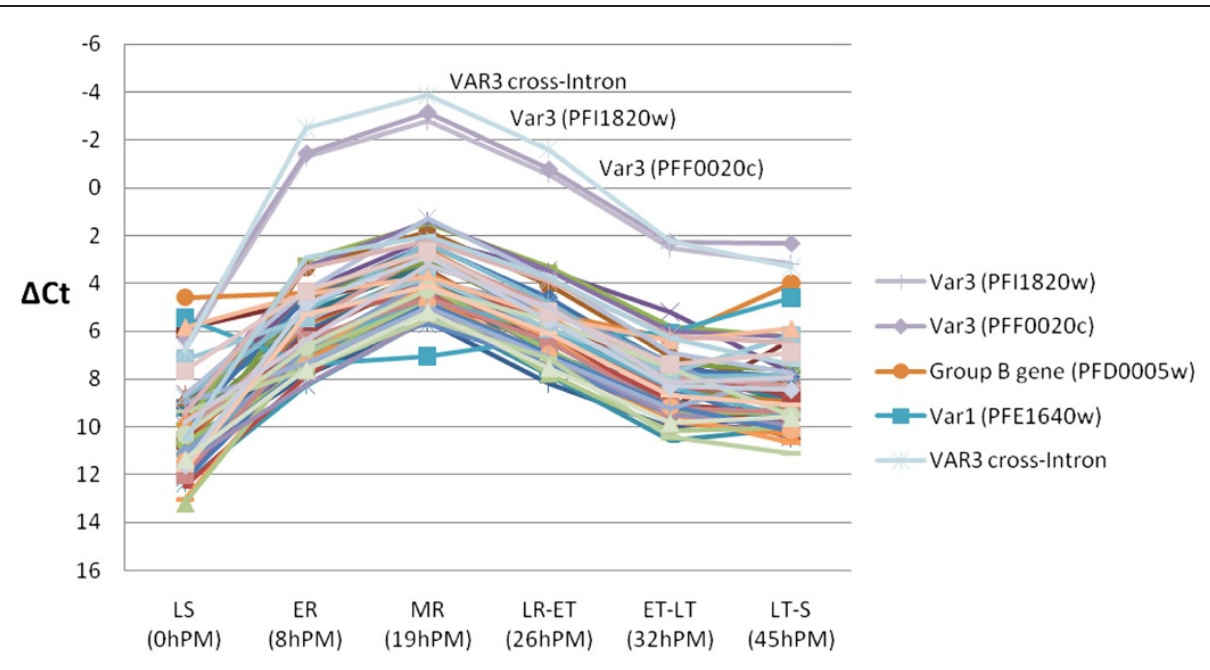

Figure 3 Transcript levels of $\mathbf{5 9}$ var genes during the intra-erythrocytic life cycle of parasite line 3D7VAR3. Transcript levels were measured by quantitative PCR and shown as amplification efficiency corrected $\triangle C t$-values relative to internal control genes. Annotations of the most prominent transcript levels are shown. LS: Late stages; ER: Early Ring; MR: Mid Ring; LR: Late Ring; ET: Early Trophozoite; LT: Late Trophozoite; S: Schizont. hPM: h post magnet-activated cell sorting. 


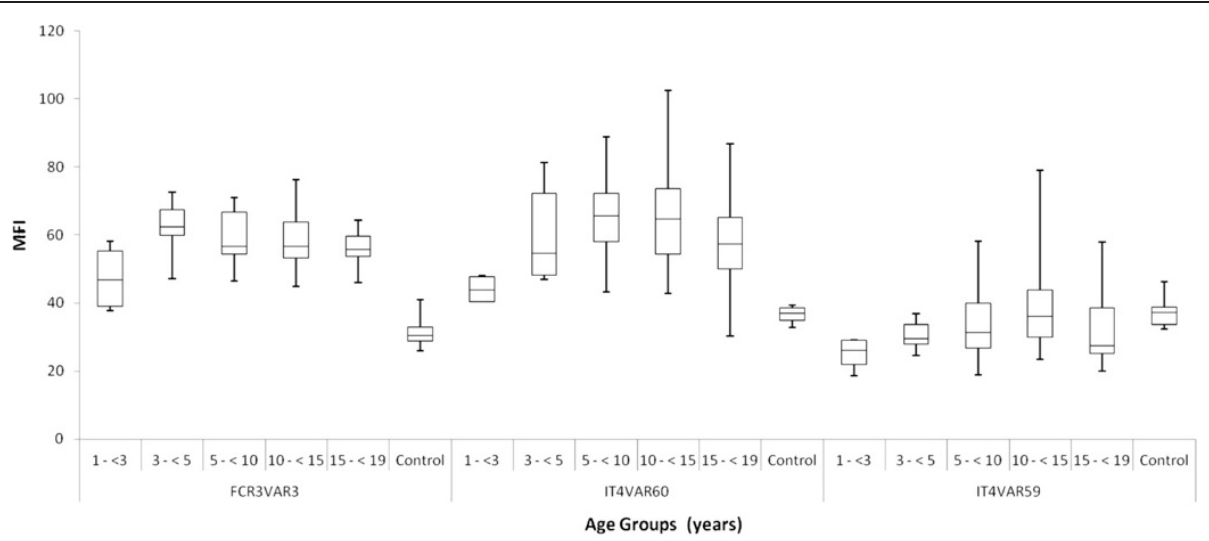

PfEMP1
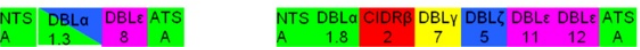

DBLa CIDRa DBLo

Figure 4 Immune serum reactivity to three IT/FCR3 Plasmodium falciparum parasite lines. The reactivity of 61 plasma samples from children living in malaria endemic Mgome, Tanzania, against three parasite lines expressing VAR3 (Group A PfEMP1), IT4var60 (Group A PfEMP1) and IT4var59 (Group B PfEMP1), respectively, was measured by flow cytometry and divided into age groups: [1- $<3] n=4$; [3- $<5] n=6$; [5- $<10]$ $n=23 ;[10-<15] n=19 ;[15-<19] n=9$. Danish controls $n=8$. The PfEMP1 domain structures as previously described [23] are shown below each parasite seroprofile. Ends of whiskers in the boxplots represent the smallest and largest median of FITC fluorescence intensity (MFI) values in the given age group.

var genes other than var3 were silenced and the var3 transcript profile during the intra-erythrocytic life cycle was similar to that of other var genes in other parasite lines $[43,52]$ and predicts as expected the surface expression of VAR3 in early trophozoite stages.
Also the VAR3 expression on the erythrocyte surface appeared similar to that of other PfEMP1. Antibodies raised towards a recombinant 3D7 VAR3 variant successfully selected VAR3 erythrocyte surface-expressing parasites from both 3D7 and IT/FCR3 parasite
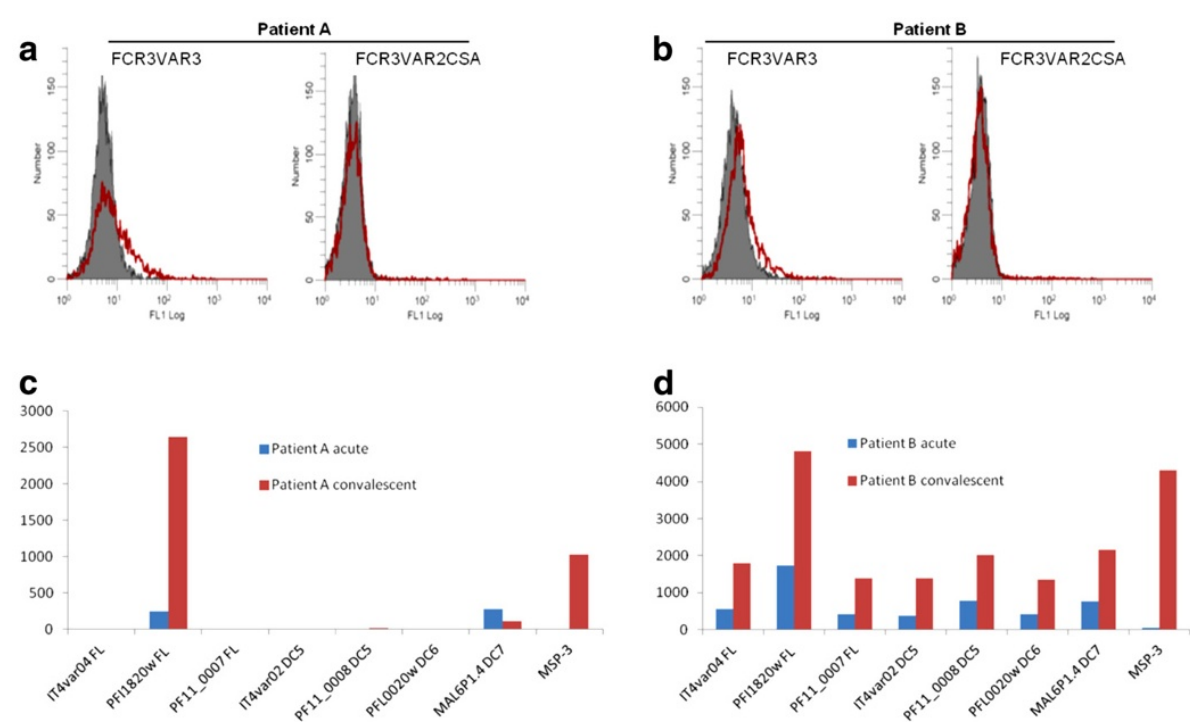

Figure 5 Reactivity to VAR3-expressing Plasmodium falciparum parasites after a malaria episode. The recognition of two $P$. falciparum parasite lines IT/FCR3 expressing VAR3 and VAR2CSA $(a, b)$ and recombinant proteins $(c, d)$ with plasma from patient $A(a, c)$ and $B(b, d)$ during a malaria episode (acute, grey shades) and after recovery (convalescent, red lines). The reactivity is shown as number of events/P. falciparum-infected erythrocytes vs. FITC flourescence intensity by flow cytometry. The acquisition of lgG to seven multi-domain and full length (FL) PfEMP1 proteins and MSP3 antigen of $P$. falciparum was measured by bead-based Luminex technology as previously described [48]. Results are expressed as median fluorescent intensity (MFI) subtracted the reactivity of unexposed control donors. The PfEMP1 domains representing domain cassette (DC) 5, 6 and 7 are as defined by Rask et al. [23]. 
populations. The surface expression of VAR3 was confirmed by antibody staining in both flow cytometry and confocal microscopy with expression levels and a punctuate pattern characteristic for PfEMP1 expression in knobs. While the VAR3 in the IT/FCR3 line most likely is the only PfEMP1 on the infected erythrocyte surface, it is possible that both PFI1820w and PFF0020c VAR3 PfEMP1s are expressed by the same parasites, as previously seen in 3D7 parasites [11].

\section{Conclusions}

In conclusion, var3 is transcribed and the encoded protein expressed on the surface of infected erythrocytes in the same manner as seen for other viable var genes. VAR3expressing parasites sustain $P$. falciparum infections in children living in malaria-endemic areas although only found in a fraction of malaria hospitalizations and not associated with severe anaemia or cerebral malaria syndromes. Whether this structurally unique and conserved PfEMP1 confer parasites expressing it with a rare functional specialization remains unclear, but further studies of the cyto-adherence phenotype of the established VAR3expressing parasite lines may elucidate this.

\section{Additional files}

Additional file 1: Specific var gene primers used for Q-RT PCR.

Additional file 2: Primers used for Plasmodium falciparum erythrocyte protein 1 full length (FL) and multi-domain expression.

Additional file 3: Var gene transcript levels of Plasmodium falciparum IT4var59 parasites. Var gene transcript levels of IT4var59 parasites relative to the average of two internal control genes. The average of control gene transcript levels is set to 100. The distribution of var transcripts is also shown (circular diagram). Transcript levels were measured by quantitative PCR using a set of primers that amplify 56 var genes from clone IT/FCR3. The grouping of var genes are as previously described $[16,17,23]$

Additional file 4: Transcript levels of var3 in patient parasite isolates. Transcript levels of var3-DBLE8 in P. falciparum parasites isolated from 130 hospitalized children (little box; red dots: patient A and B) and the var subtype transcript levels in patient parasite isolates, A and B. The levels are relative to the average of two internal control genes, which are normalized to 100 . Transcript levels were measured by quantitative PCR using a set of 42 subtyping quantitative primers [37].

\section{Competing interests}

The authors declare that they have no competing interests.

\section{Acknowledgements}

We sincerely thank the children participating in this study and parents or guardians, including teams, which conducted studies in Mgome, Muheza and Korogwe, Tanzania. We thank Ulla Abildtrup for the consistent testing of isolate integrity of cultured parasites and Susanne Lücking Nielsen for excellent technical assistance. We thank Dr Ali Salanti for supplying the full length VAR2CSA protein. CWW was supported by the The Danish Medical Research Council (grant reference number 271-08-0540) and Augustinus Fonden. LT received support University of Copenhagen Program of Excellence. TL was supported by the Grand Challenges in Global Health program, Lundbeck Foundation and The Danish Medical Research Council.

\section{Author details}

${ }^{1}$ Centre for Medical Parasitology at Department of International Health, Immunology, and Microbiology, University of Copenhagen and at Department of Infectious Diseases, Copenhagen University Hospital (Rigshospitalet), 1014 Copenhagen, Denmark. ${ }^{2}$ National Institute for Medical Research (NIMR), Tanga Medical Research Centre, Tanga, Tanzania.

\section{Authors' contributions}

CWW carried out molecular biology studies, analysed data and wrote the paper. TL and TGT participated in the design, coordination and analysis of the study and helped to draft the manuscript. LT designed and carried out molecular biology studies, analysed data and helped to draft the manuscript. DCB, SBS, AMM and MA carried out molecular biology studies and revised the manuscript. PAM and JPL participated in the coordination of the study and revised the manuscript. All authors read and approved the final manuscript.

Received: 11 February 2012 Accepted: 25 April 2012

Published: 25 April 2012

\section{References}

1. Bull PC, Lowe BS, Kortok M, Molyneux CS, Newbold Cl, Marsh K: Parasite antigens on the infected red cell surface are targets for naturally acquired immunity to malaria. Nat Med 1998, 4:358-360.

2. Bull PC, Lowe BS, Kortok M, Marsh K: Antibody recognition of Plasmodium falciparum erythrocyte surface antigens in Kenya: evidence for rare and prevalent variants. Infect Immun 1999, 67:733-739.

3. Giha HA, Staalsoe T, Dodoo D, Roper C, Satti GM, Arnot DE, Hviid L, Theander TG: Antibodies to variable Plasmodium falciparum-infected erythrocyte surface antigens are associated with protection from novel malaria infections. Immunol Lett 2000, 71:117-126.

4. Baruch DI, Pasloske BL, Singh HB, Bi X, Ma XC, Feldman M, Taraschi TF, Howard RJ: Cloning the $P$. falciparum gene encoding PfEMP1, a malarial variant antigen and adherence receptor on the surface of parasitized human erythrocytes. Cell 1995, 82:77-87.

5. Smith JD, Chitnis CE, Craig AG, Roberts DJ, Hudson-Taylor DE, Peterson DS, Pinches R, Newbold Cl, Miller LH: Switches in expression of Plasmodium falciparum var genes correlate with changes in antigenic and cytoadherent phenotypes of infected erythrocytes. Cell 1995, 82:101-110.

6. Su XZ, Heatwole VM, Wertheimer SP, Guinet F, Herrfeldt JA, Peterson DS, Ravetch JA, Wellems TE: The large diverse gene family var encodes proteins involved in cytoadherence and antigenic variation of Plasmodium falciparum-infected erythrocytes. Cell 1995, 82:89-100.

7. Chen Q, Fernandez V, Sundstrom A, Schlichtherle M, Datta S, Hagblom P, Wahlgren M: Developmental selection of var gene expression in Plasmodium falciparum. Nature 1998, 394:392-395.

8. Scherf A, Hernandez-Rivas R, Buffet P, Bottius E, Benatar C, Pouvelle B, Gysin $J$, Lanzer M: Antigenic variation in malaria: in situ switching, relaxed and mutually exclusive transcription of var genes during intra-erythrocytic development in Plasmodium falciparum. EMBO J 1998, 17:5418-5426.

9. Dzikowski R, Frank M, Deitsch K: Mutually exclusive expression of virulence genes by malaria parasites is regulated independently of antigen production. PLOS Pathog 2006, 2:e22.

10. Voss TS, Healer J, Marty AJ, Duffy MF, Thompson JK, Beeson JG, Reeder JC, Crabb BS, Cowman AF: A var gene promoter controls allelic exclusion of virulence genes in Plasmodium falciparum malaria. Nature 2006, 439:1004-1008.

11. Joergensen L, Bengtsson DC, Bengtsson A, Ronander E, Berger SS, Turner L, Dalgaard MB, Cham GK, Victor ME, Lavstsen T, Theander TG, Arnot DE, Jensen AT: Surface co-expression of two different PfEMP1 antigens on single Plasmodium falciparum-infected erythrocytes facilitates binding to ICAM1 and PECAM1. PLOS Pathog 2010, 6:e1001083.

12. Howard RJ: Malarial proteins at the membrane of Plasmodium falciparuminfected erythrocytes and their involvement in cytoadherence to endothelial cells. Prog Allergy 1988, 41:98-147.

13. Kyes $S$, Horrocks $P$, Newbold C: Antigenic variation at the infected red cell surface in malaria. Annu Rev Microbiol 2001, 55:673-707.

14. Barry AE, Trieu A, Fowkes FJ, Pablo J, Kalantari-Dehaghi M, Jasinskas A, Tan X, Kayala MA, Tavul L, Siba PM, Day KP, Baldi P, Felgner PL, Doolan DL: The stability and complexity of antibody responses to the major surface 
antigen of Plasmodium falciparum are associated with age in a malaria endemic area. Mol Cell Proteomics 2011, 10:M111.

15. Gardner MJ, Hall N, Fung E, White O, Berriman M, Hyman RW, Carlton JM, Pain A, Nelson KE, Bowman S, Paulsen IT, James K, Eisen JA, Rutherford K, Salzberg SL, Craig A, Kyes S, Chan MS, Nene V, Shallom SJ, Suh B, Peterson J, Angiuoli S, Pertea M, Allen J, Selengut J, Haft D, Mather MW, Vaidya AB, Martin DM Fairlamb AH, Fraunholz MJ, Roos DS, Ralph SA, McFadden Gl, Cummings LM, Subramanian GM, Mungall C, Venter JC, Carucci DJ, Hoffman SL, Newbold C, Davis RW, Fraser CM, Barrel B: Genome sequence of the human malaria parasite Plasmodium falciparum. Nature 2002, 419:498-511.

16. Kraemer SM, Smith JD: Evidence for the importance of genetic structuring to the structural and functional specialization of the Plasmodium falciparum var gene family. Mol Microbiol 2003, 50:1527-1538.

17. Lavstsen T, Salanti A, Jensen AT, Arnot DE, Theander TG: Sub-grouping of Plasmodium falciparum 3D7 var genes based on sequence analysis of coding and non-coding regions. Malar J 2003, 2:27.

18. Salanti A, Jensen AT, Zornig HD, Staalsoe $T$, Joergensen $L$, Nielsen MA, Khattab A, Arnot DE, Klinkert MQ, Hviid L, Theander TG: A sub-family of common and highly conserved Plasmodium falciparum var genes. Mol Biochem Parasitol 2002, 122:111-115.

19. Rowe JA, Kyes SA, Rogerson SJ, Babiker HA, Raza A: Identification of a conserved Plasmodium falciparum var gene implicated in malaria in pregnancy. J Infect Dis 2002, 185:1207-1211.

20. Salanti A, Staalsoe T, Lavstsen T, Jensen AT, Sowa MP, Arnot DE, Hviid $L$, Theander TG: Selective upregulation of a single distinctly structured var gene in chondroitin sulphate A-adhering Plasmodium falciparum involved in pregnancy-associated malaria. Mol Microbiol 2003, 49:179-191.

21. Trimnell AR, Kraemer SM, Mukherjee S, Phippard DJ, Janes JH, Flamoe E, Su $X Z$, Awadalla P, Smith JD: Global genetic diversity and evolution of var genes associated with placental and severe childhood malaria. Mol Biochem Parasitol 2006, 148:169-180.

22. Sander AF, Salanti A, Lavstsen T, Nielsen MA, Magistrado P, Lusingu J, Ndam NT, Arnot DE: Multiple var2csa-type PfEMP1 genes located at different chromosomal loci occur in many Plasmodium falciparum isolates. PLoS One 2009, 4:e6667.

23. Rask TS, Hansen DA, Theander TG, Gorm PA, Lavstsen T: Plasmodium falciparum erythrocyte membrane protein 1 diversity in seven genomes-divide and conquer. PLOS Comput Biol 2010, 6:e1000933.

24. Montgomery J, Mphande FA, Berriman M, Pain A, Rogerson SJ, Taylor TE, Molyneux ME, Craig A: Differential var gene expression in the organs of patients dying of falciparum malaria. Mol Microbiol 2007, 65:959-967.

25. Bull PC, Kortok M, Kai O, Ndungu F, Ross A, Lowe BS, Newbold Cl, Marsh K: Plasmodium falciparum-infected erythrocytes: agglutination by diverse Kenyan plasma is associated with severe disease and young host age. $J$ Infect Dis 2000, 182:252-259.

26. Nielsen MA, Staalsoe T, Kurtzhals JA, Goka BQ, Dodoo D, Alifrangis M Theander TG, Akanmori BD, Hviid L: Plasmodium falciparum variant surface antigen expression varies between isolates causing severe and nonsevere malaria and is modified by acquired immunity. $J$ Immunol 2002, 168:3444-3450

27. Bull PC, Berriman M, Kyes S, Quail MA, Hall N, Kortok MM, Marsh K, Newbold $\mathrm{Cl}$ : Plasmodium falciparum variant surface antigen expression patterns during malaria. PLOS Pathog 2005, 1:e26

28. Jensen AT, Magistrado P, Sharp S, Joergensen L, Lavstsen T, Chiucchiuini A, Salanti A, Vestergaard LS, Lusingu JP, Hermsen R, Sauerwein R, Christensen J, Nielsen MA, Hviid L, Sutherland C, Staalsoe T, Theander TG: Plasmodium falciparum associated with severe childhood malaria preferentially expresses PfEMP1 encoded by group A var genes. J Exp Med 2004, 199:1179-1190.

29. Rottmann M, Lavstsen T, Mugasa JP, Kaestli M, Jensen AT, Muller D, Theander T, Beck HP: Differential expression of var gene groups is associated with morbidity caused by Plasmodium falciparum infection in Tanzanian children. Infect Immun 2006, 74:3904-3911.

30. Falk N, Kaestli M, Qi W, Ott M, Baea K, Cortes A, Beck HP: Analysis of Plasmodium falciparum var genes expressed in children from Papua New Guinea. J Infect Dis 2009, 200:347-356

31. Lusingu JP, Jensen AT, Vestergaard LS, Minja DT, Dalgaard MB, Gesase S, Mmbando BP, Kitua AY, Lemnge MM, Cavanagh D, Hviid L, Theander TG Levels of plasma immunoglobulin $G$ with specificity against the cysteine-rich interdomain regions of a semiconserved Plasmodium falciparum erythrocyte membrane protein 1, VAR4, predict protection against malarial anemia and febrile episodes. Infect Immun 2006, 74:2867-2875

32. Magistrado PA, Lusingu J, Vestergaard LS, Lemnge M, Lavstsen T, Turner $L$, Hviid L, Jensen AT, Theander TG: Immunoglobulin $G$ antibody reactivity to a group A Plasmodium falciparum erythrocyte membrane protein 1 and protection from P. falciparum malaria. Infect Immun 2007, 75:2415-2420.

33. Kaestli M, Cockburn IA, Cortes A, Baea K, Rowe JA, Beck HP: Virulence of malaria is associated with differential expression of Plasmodium falciparum var gene subgroups in a case-control study. J Infect Dis 2006 , 193:1567-1574.

34. Kyriacou HM, Stone GN, Challis RJ, Raza A, Lyke KE, Thera MA, Kone AK, Doumbo OK, Plowe CV, Rowe JA: Differential var gene transcription in Plasmodium falciparum isolates from patients with cerebral malaria compared to hyperparasitaemia. Mol Biochem Parasitol 2006, 150:211-218.

35. Normark J, Nilsson D, Ribacke U, Winter G, Moll K, Wheelock CE, Bayarugaba J, Kironde F, Egwang TG, Chen Q, Andersson B, Wahlgren M: PfEMP1DBL1alpha amino acid motifs in severe disease states of Plasmodium falciparum malaria. Proc Natl Acad Sci USA 2007, 104:15835-15840.

36. Warimwe GM, Keane TM, Fegan G, Musyoki JN, Newton CR, Pain A, Berriman M, Marsh K, Bull PC: Plasmodium falciparum var gene expression is modified by host immunity. Proc Natl Acad Sci USA 2009, 106:21801-21806.

37. Lavstsen T, Turner L, Saguti F, Magistrado P, Rask TS, Jespersen JS, Wang CW Berger SS, Baraka V, Marquard AM, Seguin-Orlando A, Willerslev E, Gilbert TP, Lusingu J, Theander TG: $P$. falciparum erythrocyte membrane protein 1 domain cassettes 8 and 13 are associated with severe malaria in children. Proc Natl Acad Sci USA, in press.

38. Cham GK, Turner L, Lusingu J, Vestergaard L, Mmbando BP, Kurtis JD, Jensen AT, Salanti A, Lavstsen T, Theander TG: Sequential, ordered acquisition of antibodies to Plasmodium falciparum erythrocyte membrane protein 1 domains. J Immunol 2009, 183:3356-3363.

39. Epp C, Li F, Howitt CA, Chookajorn T, Deitsch KW: Chromatin associated sense and antisense noncoding RNAs are transcribed from the var gene family of virulence genes of the malaria parasite Plasmodium falciparum. RNA 2009, 15:116-127.

40. Cranmer SL, Magowan C, Liang J, Coppel RL, Cooke BM: An alternative to serum for cultivation of Plasmodium falciparum in vitro. Trans $R$ Soc Trop Med Hyg 1997, 91:363-365.

41. Staalsoe T, Nielsen MA, Vestergaard LS, Jensen AT, Theander TG, Hviid L: In vitro selection of Plasmodium falciparum 3D7 for expression of variant surface antigens associated with severe malaria in African children. Parasite Immunol 2003, 25:421-427.

42. Snounou G, Zhu X, Siripoon N, Jarra W, Thaithong S, Brown KN, Viriyakosol S: Biased distribution of msp1 and msp2 allelic variants in Plasmodium falciparum populations in Thailand. Trans R Soc Trop Med Hyg 1999, 93:369-374.

43. Dahlback M, Lavstsen T, Salanti A, Hviid L, Arnot DE, Theander TG, Nielsen MA: Changes in var gene mRNA levels during erythrocytic development in two phenotypically distinct Plasmodium falciparum parasites. Malar $\mathrm{J}$ 2007, 6:78.

44. The Wellcome Trust Sanger Institute (UK) [www.sanger.ac.uk/Projects/ P falciparum]

45. Wang CW, Magistrado PA, Nielsen MA, Theander TG, Lavstsen T: Preferential transcription of conserved rif genes in two phenotypically distinct Plasmodium falciparum parasite lines. Int J Parasitol 2009, 39:655-664

46. Heegaard ED, Qvortrup K, Christensen J: Baculovirus expression of erythrovirus V9 capsids and screening by ELISA: serologic cross-reactivity with erythrovirus B19. J Med Virol 2002, 66:246-252.

47. Khunrae P, Dahlback M, Nielsen MA, Andersen G, Ditlev SB, Resende M, Pinto W, Theander TG, Higgins MK, Salanti A: Full-length recombinant Plasmodium falciparum VAR2CSA binds specifically to CSPG and induces potent parasite adhesion-blocking antibodies. J Mol Biol 2010, 397:826-834.

48. Turner L, Wang CW, Lavstsen T, Mwakalinga SB, Sauerwein RW, Hermsen CC, Theander TG: Antibodies against PfEMP1, RIFIN, MSP3 and GLURP are acquired during controlled Plasmodium falciparum malaria infections in naive volunteers. PLOS One 2011, 6:e29025.

49. Staalsoe T, Giha HA, Dodoo D, Theander TG, Hviid L: Detection of antibodies to variant antigens on Plasmodium falciparum-infected erythrocytes by flow cytometry. Cytometry 1999, 35:329-336. 
50. Lusingu JPA, Vestergaard LS, Mmbando BP, Drakeley CJ, Jones C, Akida J, Savaeli ZX, Kitua AY, Lemnge MM, Theander TG: Malaria morbidity and immunity among residents of villages with different Plasmodium falciparum transmission intensity in North-Eastern Tanzania. Malar J 2004, 3:26.

51. Bengtsson D, Sowa KM, Salanti A, Jensen AT, Joergensen $L$, Turner $L$, Theander TG, Arnot DE: A method for visualizing surface-exposed and internal PfEMP1 adhesion antigens in Plasmodium falciparum infected erythrocytes. Malar J 2008, 7:101.

52. Cham GK, Kurtis J, Lusingu J, Theander TG, Jensen AT, Turner L: A semi-automated multiplex high-throughput assay for measuring $\lg G$ antibodies against Plasmodium falciparum erythrocyte membrane protein 1 (PfEMP1) domains in small volumes of plasma. Malar $J$ 2008, 7:108.

53. Tuikue Ndam NG, Salanti A, Bertin G, Dahlback M, Fievet N, Turner L, Gave A, Theander T, Deloron P: High level of var2csa transcription by Plasmodium falciparum isolated from the placenta. I Infect Dis 2005, 192:331-335.

54. Albrecht L, Moll K, Blomqvist K, Normark J, Chen Q, Wahlgren M: var gene transcription and PfEMP1 expression in the rosetting and cytoadhesive Plasmodium falciparum clone FCR3S1.2. Malar J 2011, 10:17.

55. Kraemer SM, Smith JD: A family affair: var genes, PfEMP1 binding, and malaria disease. Curr Opin Microbiol 2006, 9:374-380.

56. Kyes SA, Christodoulou Z, Raza A, Horrocks P, Pinches R, Rowe JA, Newbold Cl: A well-conserved Plasmodium falciparum var gene shows an unusual stage-specific transcript pattern. Mol Microbiol 2003, 48:1339-1348.

doi:10.1186/1475-2875-11-129

Cite this article as: Wang et al.: Evidence for in vitro and in vivo expression of the conserved VAR3 (type 3) plasmodium falciparum erythrocyte membrane protein 1. Malaria Journal 2012 11:129.

\section{Submit your next manuscript to BioMed Central and take full advantage of:}

- Convenient online submission

- Thorough peer review

- No space constraints or color figure charges

- Immediate publication on acceptance

- Inclusion in PubMed, CAS, Scopus and Google Scholar

- Research which is freely available for redistribution 